\title{
Association between toluene diisocyanate-induced asthma and DQB1 markers: a possible role for aspartic acid at position 57
}

\author{
A. Balboni*, O.R. Baricordi*, L.M. Fabbri**, E. Gandini*, A. Ciaccia**, C.E. Mapp ${ }^{+}$
}

\begin{abstract}
Association between toluene diisocyanate-induced asthma and DQB1 markers: a possible role for aspartic acid at position 57. A. Balboni, O.R. Baricordi, L.M. Fabbri, E. Gandini, A. Ciaccia, C.E. Mapp. (C) ERS Journals Ltd 1996.

ABSTRACT: Toluene diisocyanate (TDI) is the most common cause of occupational asthma in western countries. The aim of this study was to investigate whether genetic factors are involved in toluene diisocyanate-induced asthma.

We studied the frequency of human leucocyte antigen (HLA) class II genetic markers in three groups of subjects: 1) subjects with TDI-induced asthma $(n=30)$; 2) exposed subjects with no history of TDI-induced asthma $(\mathrm{n}=12)$; and 3$)$ normal subjects not exposed to TDI $(n=126)$. Venous blood samples were collected from the three groups and the polymorphic second exon of DQA and DQB genes was amplified by the polymerase chain reaction (PCR) method.

Evaluation of HLA class II gene products in TDI-induced asthma cases showed a positive association with HLA-DQB1*0503 and a negative association with HLADQB1*0501 alleles, which differed at residue 57 for a single amino acid, i.e. aspartic acid in DQB1*0503 and valine in DQB1*0501. No significant difference was found in the distribution of DQA1 alleles between asthmatics and controls.

Our results confirm the hypothesis that HLA-DQB1*0503 has a role in conferring susceptibility to TDI-induced asthma and that residue 57 of HLA-DQB1 is a potentially critical location.
\end{abstract}

Eur Respir J., 1996, 9, 207-210.

*Institute of Medical Genetics and **Institute of Pulmonary Diseases, University of Ferrara, Italy. +Institute of Occupational Medicine, University of Padova, Italy.

Correspondence: C.E. Mapp,

Istituto di Medicina del Lavoro

Via J Facciolati 71

35127 Padova

Italy

\section{Keywords: Asthma \\ human leucocyte antigen isocyanates}

Received: February 131995 Accepted after revision October 111995

Supported by the National Research Council (FATMA 93.00650.PF41), the MURST (40\% and 60\%), the Regione Veneto, and Regione Emilia-Romagna (Ricerca Sanitaria Finalizzata).

Diisocyanates are used worldwide in a number of important industries. They are a group of low molecular weight chemical compounds characterized by the presence of an $-\mathrm{N}=\mathrm{C}=\mathrm{O}$ group. The most important compound is toluene diisocyanate (the 80:20 mixture of the two isomers 2,4 and 2,6). Exposure to diisocyanates is now recognized as a leading cause of occupational asthma [1]. It is generally accepted that $5 \%$ of exposed subjects develop occupational asthma after exposure to toluene diisocyanate (TDI) [2], but estimates as high as $15 \%$ have been proposed [3]. The mechanism of TDI-induced asthma is still unknown but recent evidence suggests an immunological mechanism [4]. In fact, TDI-induced asthma and extrinsic asthma have a similar pattern of inflammatory cell infiltration in bronchial biopsies $[5,6]$; inhalation challenge with TDI causes an increase in circulating CD8+ lymphocytes and eosinophils in sensitized subjects [7]; and an association with human leucocyte antigen (HLA) class II genes is documented both in TDIinduced asthma [8] and in allergen-induced asthma [9, 10].

In the present study, we investigated whether HLA class II genes are involved in TDI-induced asthma by studying the distribution of the markers DQA and DQB in subjects with TDI-induced asthma, in asymptomatic subjects exposed to isocyanates and in healthy subjects not exposed to isocyanates.

\begin{abstract}
Methods
The study was carried out according to the principles of the Declaration of Helsinki, with written informed consent.
\end{abstract}

\section{Subjects}

All subjects were European-born, unrelated whites working in the north of Italy. The 30 subjects with TDIinduced asthma had documented asthma. Diagnosis of occupational asthma was based on the following: exposure to isocyanates; asthma symptoms; airway hyperresponsiveness to methacholine inhalation challenge; an asthmatic reaction (fall in forced expiratory volume in one second (FEV1) of at least 20\%) after exposure to TDI in the laboratory. Twelve asymptomatic subjects exposed for a long period to isocyanates were also studied. For ethical reasons, they were not submitted to inhalation challenge with methacholine or TDI. One hundred and twenty six healthy subjects not exposed to isocyanates were also studied, selected from a panel of unrelated Italian blood donors. The characteristics of occupational asthmatics and control exposed subjects are shown in table 1 . 
Table 1. - Characteristics of subjects with isocyanateinduced asthma and of control asymptomatic exposed subjects

\begin{tabular}{lcc}
\hline & Asthmatics & Controls \\
$\mathrm{n}$ & 30 & 12 \\
\hline Sex M/F & $23 / 7$ & $12 / 0$ \\
Age yrs & $40 \pm 2$ & $45 \pm 2$ \\
Smoking habit NS/S/EX & $22 / 4 / 4$ & $4 / 7 / 1$ \\
Exposure to TDI yrs & $14.7 \pm 1.9$ & $15.8 \pm 3.0$ \\
Onset of asthma yrs & $5.5 \pm 0.8$ & \\
Baseline FEV1 \% pred & $99 \pm 3$ & $107 \pm 3$ \\
\hline
\end{tabular}

Values are expressed as mean \pm SEM. ${ }^{*}$ : predicted values from [23]. NS: nonsmokers; S: smokers; EX: ex-smokers; M: male; F: female; TDI: toluene diisocyanate; FEV1: forced expiratory volume in one second.

\section{Deoxyribonucleic acid (DNA) preparation}

Blood samples $(20 \mathrm{~mL})$ were obtained from all subjects. Genomic DNA was extracted from peripheral whole blood using the classic phenol chloroform method [11].

\section{Polymerase chain reaction $(P C R)$}

The polymorphic second exon of DQA and DQB genes was selectively amplified by the polymerase chain reaction (PCR) method using XI International Histocompatibility Workshop primers [12]. One microgram of DNA of each sample was amplified in a $50 \mu \mathrm{L}$ reaction volume, according to XI International Histocompatibility Workshop conditions. The efficiency of amplification was controlled by resolving $5 \mu \mathrm{L}$ PCR product electrophoresis on a $1.5 \%$ agarose gel as a 229 base pair (bp) band for DQA and a 214 bp band for DQB after ethidium staining.

\section{Analysis of the PCR products}

Three microlitres of each PCR product were denatured in $0.4 \mathrm{~N} \mathrm{NaOH}, 25 \mathrm{mM}$ ethylenediamine tetra-acetic acid (EDTA) and dot-blotted on a nylon membrane (Bio-Rad Zeta Probe) using a Bio-Dot Apparatus (Bio-Rad). Nonradioactive $3^{\prime}$ end-labelling of the selected probes was performed with digoxigenin-11-2'-3'-dideoxyuridine-5'triphosphate (Dig-11-ddUTP; Boehringer Mannheim $\mathrm{GmbH}$, Germany) and DNA deoxynucleotidyl transferase (Terminal Transferase), Boehringer Mannheim $\mathrm{GmbH}$, Germany) at $37^{\circ} \mathrm{C}$ for $15 \mathrm{~min}$. To make hybridization conditions independent of G-C content, tetramethylammonium chloride (TMACl) solution [12] was used in order to perform washing [13]. Chemiluminescent detection of the probes was performed by incubating nylon membranes with $0.5 \mathrm{~mL} \cdot 100 \mathrm{~cm}^{-2}$ of $1: 100$ Lumigen PPD (Boehringer Mannheim $\mathrm{GmbH}$, Germany) at $37^{\circ} \mathrm{C}$ for $15 \mathrm{~min}$, with membranes sealed in acetate bags. Sealed membranes were then exposed to Kodak X-OMAT film with intensifying screens for 20-30 min at room temperature [12]. Evaluation of positivities on each film was performed blind to clinical status.

\section{HLA typing}

Twelve probes for DQAl and 15 probes for DQB1 molecular typing were selected from all the DQ probes proposed by the XI Histocompatibility Workshop. A total of eight DQA1 and 14 DQB1 alleles could be identified. DQA and DQB molecular typing of eight homozygous cell lines of known specificities was performed as control.

\section{Statistical analysis}

Fisher's exact test was utilized. Relative risk (RR) was calculated by using the Wolf method with Haldane's modification [14]. Probability values were corrected for number of comparisons according to Bonferroni's rule, taking into account alleles previously reported as associated to TDI-induced asthma, the p-values obtained were corrected $(\mathrm{pc})$ only multiplying for the number of a priori hypotheses [15].

\section{Results}

Of the 30 asthmatic subjects, 23 were males and 7 females (table 1). Age (mean \pm SEM) was $40 \pm 2$ yrs, length of exposure to TDI $14.7 \pm 1.9 \mathrm{yrs}$, and time since onset of asthma $5.5 \pm 0.8$ yrs. The 12 asymptomatic exposed subject were all males, exposed to TDI for $15.8 \pm 3.0 \mathrm{yrs}$.

No significant difference was found in the distribution of the DQA1 phenotypic frequencies in the asthmatic subjects when compared to both control groups (asymptomatic exposed subjects and healthy, nonexposed subjects). The comparison of DQB1 phenotypic frequencies between asthmatics and asymptomatic exposed subjects and healthy controls revealed differences in two alleles, the DQB $1 * 0501$ and the DQB $1 * 0503$ (fig. 1 ). The frequency of $\mathrm{DQB} 1 * 0503$ was $30 \%$ in the asthmatic subjects and $0 \%$ in the asymptomatic exposed subjects $(\mathrm{p}=0.032$; $\mathrm{pc}=\mathrm{NS})$. In contrast, the frequency of the DQB $1 * 0501$ marker was $0 \%$ in the asthmatic subjects and $25 \%$ in the asymptomatic exposed subjects $(\mathrm{p}=0.019 ; \mathrm{pc}=0.038$; $\mathrm{RR}=0.044)$. The frequency of the $\mathrm{DQB} 1 * 0503$ marker
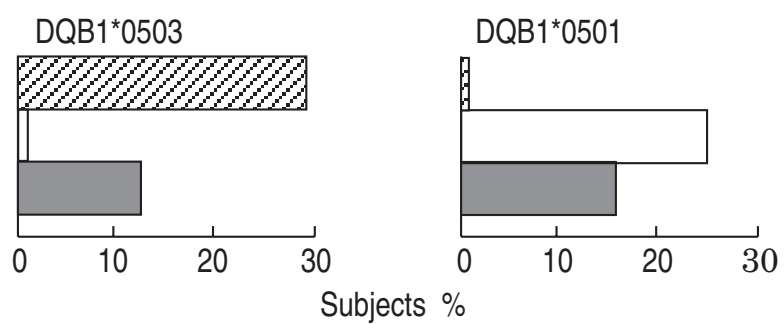

Fig. 1. - Distribution of DQB1 markers in 30 asthmatics ( $\square$ ), in 12 asymptomatic exposed controls $(\square)$, and in 126 healthy controls ( $\square$ ). DQB $1 * 0501$ : $\mathrm{p}=0.019$; $\mathrm{pc}=0.038 ; \mathrm{RR}=0.044$ (comparison between asthmatics and asymptomatic exposed controls); $\mathrm{p}=0.010$; $\mathrm{pc}=0.020 ; \mathrm{RR}=0.085$ (comparison between asthmatics and healthy controls). DQB1*0503: $\mathrm{p}=0.032$; $\mathrm{pc}=\mathrm{NS}$ (comparison between asthmatics and asymptomatic exposed controls); $\mathrm{p}=0.025 ; \mathrm{pc}=0.050$; $\mathrm{RR}=2.95$ (comparison between asthmatics and healthy controls). $\mathrm{p}$ : Fisher's probability; pc: corrected for the number of a priori hypotheses; RR: relative risk; NS: not significant. 
Table 2. $-2 \times 3$ table of distribution of DQB1Asp57+ haplotypes in 30 asthmatics and in 138 clustered controls

\begin{tabular}{lrrrrr}
\hline $\begin{array}{l}\text { DQB1Asp } \\
\text { haplotypes* }\end{array}$ & Asthmatics & \multicolumn{2}{c}{ Controls } & \\
\hline 2 & $\mathrm{n}$ & $\%$ & $\mathrm{n}$ & $\%$ & $\mathrm{p}$-value \\
1 & 17 & 57 & 44 & 32 & 0.0103 \\
0 & 12 & 40 & 73 & 53 & \\
\hline & 1 & 3 & 21 & 15 & \\
\hline
\end{tabular}

: $2 \mathrm{Asp}^{57+}$ haplotypes=homozygotes $\mathrm{Asp}^{57+}, 1 \mathrm{Asp}^{57+}$ haplotype $=$ heterozygotes $\mathrm{Asp}^{57+}, 0 \mathrm{Asp}^{57+}=$ homozygotes Asp ${ }^{57-}$. Chi-squared $=7.324,0.025<\mathrm{p}<0.05$.

was significantly higher in asthmatics (30 vs $12.7 \%$; $\mathrm{p}=0.025 ; \mathrm{pc}=0.050 ; \mathrm{RR}=2.95)$, whereas the frequency of the marker DQB $1 * 0501$ was significantly lower in asthmatics compared to healthy controls ( 0 vs $15.9 \%$; $\mathrm{p}=0.010$; $\mathrm{pc}=0.020 ; \mathrm{RR}=0.085$ ).

The nucleotide sequences of DQB1 alleles are well established [16]. The two alleles DQB $1 * 0503$ and DQB $1 * 0501$ differ in one single position, 57, with aspartic acid in DQB $1 * 0503$ and valine in DQB $1 * 0501$ allele. We hypothesized that this difference in position 57 could be important for susceptibility and protection. Therefore, we have classified the 14 DQB1 alleles, on the basis of the sequence reported in the literature [16] into DQB1Asp57+

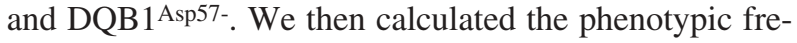
quencies of the DQB1-Asp ${ }^{57+}$ marker in the three groups. Aspartic acid was present in 29 out of the 30 subjects with isocyanate-induced asthma (97\%) compared to 105 of the 126 control subjects $(83 \%)(\mathrm{p}=0.0447 ; \mathrm{RR}=5.8)$, whilst all 12 of the asymptomatic exposed control subjects carried at least one DQB1-Asp ${ }^{57+}$ haplotype.

Because of the suggested functional implications of the presence of aspartic acid at position 57 in the DQ beta chain, we suspected a gene dose effect. Therefore, we analysed the distribution of the DQB1-Asp57+ haplotypes in the different populations, comparing the number of homozygotes and heterozygotes in asthmatics with those in controls. Since the distribution of DQB1-Asp ${ }^{57+}$ haplotypes was not significantly different in the asymptomatic exposed subjects and in the healthy controls, we clustered the data of these two groups. We found a significant difference between the distribution of homozygote and heterozygote subjects for Asp ${ }^{57+}$ haplotypes in the two populations with a p-value near 0.025 , suggesting a gene dose effect in predisposition to isocyanateinduced asthma (table 2).

\section{Discussion}

Recently, Bignon et al. [8] have shown that HLA class II alleles are involved in isocyanate-induced asthma, with allele DQB1*0503 and the allelic combination DQB1*0201/0301 associated with susceptibility and allele DQB $1 * 0501$ and the DQA1*0101-DQB1*0501-DR1 haplo-type associated with protection against the disease. In the present study, in a different group of subjects, by using different techniques and by performing the statistical testing at the phenotype level [15], we confirmed that the HLA-DQB1 marker has a role in the disease. The two markers
DQB1*0503 and DQB1*0501 involved in susceptibility and in protection differ for a single amino acid at position 57, with aspartic acid in the DQB $1 * 0503$ and valine in the DQB1*0501 marker [16]. Aspartic acid residue was present in homozygotic condition in the majority of subjects with isocyanate-induced asthma, suggesting a direct role of the residue at position 57 in the aetiology of TDI-induced asthma.

The study by BIGNON et al. [8] and the present results are consistent with the hypothesis that an immunological mechanism is involved in TDI-induced asthma and that specific genetic factors play a role in conferring susceptibility to or protection against this disease. An immunological mechanism is likely to occur in TDIinduced asthma since the inflammatory response of the airways in TDI-induced asthma is characterized not only by increased numbers of mucosal eosinophils and mast cells but also by persistent activation of lymphocytes and by chronic expression of proinflammatory cytokines [17]. When associations between HLA class II genes and occupational asthma are investigated, the problems encountered in the field of atopic diseases are less important [18]. In fact, the phenotype of occupational asthma due to exposure to TDI may be defined with accuracy [19].

In this study, we found a positive and a negative association with two HLA class II markers. When we analysed the differences between hypervariable amino acid residues in the DQB1 alleles, we found that residue 57 of HLADQB1 has a role in conferring susceptibility to the disease. It is well-established that HLA class II molecules are involved in antigen presentation and that a correct binding between these glycoproteins and the antigen is necessary for a functional antigen recognition by $\mathrm{T}$ lymphocytes.

We do not know what the TDI antigen looks like. It seems unlikely that TDI itself is the antigen, since it is a low molecular weight chemical (MW=174.2). In animal studies, it has been shown that inhaled radiolabelled TDI rapidly accumulates in the lung and becomes potentially accessible to immunocompetent cells [20]. We assume that TDI binds to endogenous or exogenous peptides that are recognized by the T-lymphocytes as a foreign antigen. More than one mechanism may be advanced for TDI. TDI could behave as a hapten and it could alter the structure or the specificity of the T-cell receptor (TCR) directly or by acting at the gene level, or it could react with membrane proteins, such as adhesion molecules amplifying T-cell B-cell interactions. Residue 57 is a negatively charged residue that may directly interact with TDI itself. Finally, it could modify the structure of MHC class II molecules, or self-peptides at the surface of the antigen presenting cells (APC) in such a way that the modified epitopes are recognized as foreign by the T-cells. Other possibilities include the hypothesis that, since DQ molecules act as dominant suppressor genes (Is) involved in active suppression against specific antigens [21], TDI could cause a dysfunction in the suppressor-inducer network. This behaviour is supported by the finding of an increase in circulating CD8+ lymphocytes in sensitized subjects exposed to TDI in the laboratory [7], and by the finding that T-cell clones 
obtained from endobronchial biopsies of subjects with TDI-induced asthma exhibit the CD8+ phenotype and produce interleukin-5 (IL-5) and interferon-gamma [22].

Whatever the exact mechanism of the association DQTDI-induced asthma may be, the data are consistent with the hypothesis that at least two risk factors exist for TDIinduced asthma, exposure to the chemical and inheritance of the genetic marker. We do not know how the interaction works and what proportion of subjects exposed to TDI with the marker develop the disease. What is important is that most asthmatic subjects have the marker in both haplotypes. Since asthma is likely to be a multifactorial and polygenic disease, other genes or other factors, i.e. environmental factors, may be necessary for expression of TDI-induced asthma. A multifactorial inheritance may explain the low incidence in the exposed subjects, the presence of healthy subjects carrying the predisposing marker and of asthmatics lacking the same marker. Therefore, it could be that the identification of a variability at the 57 position in the $\mathrm{DQBl}$ chain is not enough to cause the expression of the disease, and other factors, particularly environmental conditions, may be necessary, in addition to HLA gene products, to determine sensitization to a compound present in the workplace and to cause asthma.

Taken together, these results suggest a role for HLA class II genes in conferring susceptibility or protection against the disease. However, on the basis of the previous considerations, at the present moment, we do not know whether the recognition of the amino acid residue variation in the "beta chain" structure is a useful tool for secondary prevention of TDI-induced asthma.

Acknowledgements: The authors thank P. Bortolami, I Adinolfi and S. Milanetto for technical assistance, L. Zedda for drawing the figure, G. Fulgeri for typing the manuscript and $\mathrm{C}$. Howarth for editing the manuscript.

\section{References}

1. Chan-Yeung M, Malo JL. Aetiological agents in occupational asthma. Eur Respir J 1994; 7 : 346-371.

2. Butcher BT, Jones RN, O'Neil CE, et al. Longitudinal study of workers employed in the manufacture of toluene diisocyanate. Am Rev Respir Dis 1977; 116: 411-421.

3. Peters JM, Wegman DH. Epidemiology and toluene diisocyanate (TDI) induced respiratory disease. Environ Health Perspect 1975; 11: 97-100.

4. Mapp CE, Saetta M, Maestrelli P, et al. Mechanisms and pathology of occupational asthma. Eur Respir $J$ 1994; 7: 544-554.

5. Azzawi M, Bradley B, Jeffery PK, et al. Identification of activated T-lymphocytes and eosinophils in bronchial biopsies in stable atopic asthma. Am Rev Respir Dis 1990; 142: 1407-1413.

6. Bentley AM, Maestrelli P, Saetta M, et al. Activated Tlymphocytes and eosinophils in the bronchial mucosa in isocyanate-induced asthma. J Allergy Clin Immunol 1992; 89: 821-829.

7. Finotto S, Fabbri LM, Rado V, Mapp CE, Maestrelli P. Increase in numbers of CD8 positive lymphocytes and eosinophils in peripheral blood of subjects with late asthmatic reactions induced by toluene diisocyanate. $\mathrm{Br} \mathrm{J}$ Ind Med 1991; 48: 116-121.

8. Bignon JS, Aron Y, Ju LY, et al. HLA class II alleles in isocyanate-induced asthma. Am J Respir Crit Care Med 1994; 149: 71-75.

9. Marsh DG, Hsu SH, Roebber M, et al. HLD-Dw2: a genetic marker for human immune response to short ragweed pollen allergen Ra5. J Exp Med 1982; 155: 1439-1451.

10. Marsh DG. Immunogenetic and immunochemical factors determining immune responsiveness to allergens: studies in unrelated subjects. In: Marsh DG, Blumenthal MN, eds. Genetic and Environmental Factors in Clinical Allergy. Minneapolis, University of Minnesota Press, 1990; pp. 97-123.

11. Maniatis A, Fritsch EF, Sambrock J. Molecular cloning, a laboratory manual. Cold Spring Harbor Laboratory, New York, 1989.

12. Nevinny-Stickel C, Keller E, Bettinotti MDL. Nonradioactive HLA class II typing using polymerase chain reaction, digoxigeninlabelled oligonucleotides, and chemiluminescent detection. In: Tsuji K, Aizawa M, Sasazuki T, eds. HLA 1991: Proceedings of the 11th International Histocompatibility Workshop and Conference. Oxford Science publ., 1992; Vol. 2, p.302.

13. Wood WI, Gitscher J, Lasky LA, Lawn RM. Base composition-independent hybridization in tetramethylammonium chloride: a method for oligonucleotide screening of highly complex gene libraries. Proc Natl Acad Sci 1985; 82: 1585 .

14. Haldane JBS. The estimation and significance of the logarithm of a ratio of frequencies. Am Hum Genet 1956; 20: 309-311.

15. Svejgaard A, Ryder LP. HLA and disease associations: detecting the strongest association. Tissue Antigens 1994; 43: 18-27.

16. Marsh SGE, Bodmer JG. HLA class II nucleotide sequences. Tissue Antigens 1992; 40: 229-243.

17. Maestrelli P, Di Stefano A, Occari P, et al. Cytokines in the airway mucosa of subjects with asthma induced by toluene diisocyanate. Am J Respir Crit Care Med 1995; 151: 607-612.

18. Evidence for an increase in atopic disease and possible causes. Special article. Clin Exp Allergy 1993; 23: 484-492.

19. Mapp CE, Boschetto P, Dal Vecchio L, Maestrelli P, Fabbri LM. Occupational asthma due to isocyanates. Eur Respir J 1988; 1: 273-279.

20. Kennedy AL, Stock MF, Alarie Y, Brown WE. Uptake and distribution of $14 \mathrm{C}$ during and following inhalation exposure to radioactive toluene diisocyanate. Toxicol Appl Pharmacol 1989; 100: 280-292.

21. Fukunaga M, Hirayama K, Sasazuki T. Activation of human CD8+ suppressor T-cells by antigen-specific CD4+ T-cell line in vitro. Hum Immunol 1989; 25: 157-168.

22. Maestrelli P, Del Prete GF, De Carli M, et al. CD8 Tcell clones producing interleukin-5 and interferon-gamma in bronchial mucosa of patients with asthma induced by toluene diisocyanate. Scand J Work Environ Health 1994; 20: 376-381.

23. Commissions des Communautes Europennes (CECA). Tables de reference pour les examens spirometriques. Office des publications officielles des Communautes Europennes. Luxembourg, 1971. 